\title{
Serial transperineal sector prostate biopsies: impact on long-term erectile dysfunction
}

\author{
James JY Chong ${ }^{1}$, Mieke Van Hemelrijck², Declan Cahill ${ }^{3}$ and Janette Kinsella ${ }^{3}$ \\ ${ }^{1}$ Faculty of Life Sciences and Medicine, King's College London, London SE1 1UL, UK \\ 2Division of Cancer Studies, Cancer Epidemiology Group, Research Oncology, King's College London, London SE1 9RT, UK \\ ${ }^{3}$ Department of Urology, The Royal Marsden NHS Foundation Trust, London SW3 6JJ, UK
}

Correspondence to: James JY Chong. Email: james.chong@kcl.ac.uk

\begin{abstract}
We wanted to determine whether serial transperineal sector prostate biopsies have a long-term effect on erectile dysfunction (ED). A total of 64 men with prostate cancer entered our active surveillance (AS) programme after a transrectal prostate biopsy as well as a confirmatory initial transperineal sector prostate biopsy (TPSBx). A repeat TPSBx was performed 24 months later as part of our active surveillance protocol. The International Index of Erectile Function-5 (IIEF-5) questionnaire assessed ED at baseline prior to each TPSBx, and at one, three, and six months after first and second TPSBX.

There was a significant short-term deterioration in erectile function on mean IIEF-5 score between baseline (19.5), when compared to one month (10.5) $(P<0.001)$ and three months $(18.7)(P=0.001)$ following first TPSBx. This resolved at six month follow-up $(19.6)(P=0.681)$. Following second TPSBx, there was a deterioration in erectile function between baseline (16.6), compared to one month (7.3), three months (13.8), and six months (15.9) ( $P<0.05)$ following second TPSBx. Initial TPSBx caused significant short-term ED, which resolved by six months. Serial TPSBx appears to have an adverse impact on erectile function in men monitored on AS, increasing the risk of long-term ED. This risk should be highlighted and discussed during the consent process.
\end{abstract}

Keywords: prostatic neoplasms, biopsy, erectile dysfunction, urologic surgical procedures

Published: $23 / 05 / 2016$

Received: 14/01/2016

ecancer 2016, 10:643 DOI: 10.3332/ecancer.2016.643

Copyright: $\odot$ the authors; licensee ecancermedicalscience. This is an Open Access article distributed under the terms of the Creative Commons Attribution License (http://creativecommons.org/licenses/by/3.0), which permits unrestricted use, distribution, and reproduction in any medium, provided the original work is properly cited. 


\section{Introduction}

More men with newly diagnosed prostate cancer are opting to enter AS programmes which allows them to defer or avoid radical therapy if their disease is low-risk [1, 2] or until their disease profile changes. This delays the potential complications associated with treatment such as erectile dysfunction (ED). Unfortunately, some of these complications can also arise after biopsy. Prostate biopsy is used in AS to initially risk stratify men and also to serially detect any increase in volume or grade of disease.

Reports on serial transrectal prostate biopsy (TRBx) disagree on whether serial biopsies affect erectile function in men on AS [3, 4]. Transperineal sector prostate biopsy (TPSBx) helps to diagnose a more significant and previously undiagnosed cancer as compared to TRBx [5-7], and in many UK centres it is now a routine part of AS. Short-term complications of TPSBx typically include haematuria, acute urinary retention, and dysuria [5,8]. There have been relatively few reports on ED after serial sector biopsies performed via the transperineal route [9].

As men are likely to have more than one TPSBx during their AS monitoring, it is important to ensure that possible long-term complications of AS are accurately identified. We wanted here to determine whether serial TPSBx have a long-term effect on ED.

\section{Materials and methods}

The current study consists of a consecutive series of 64 men who underwent TRBx followed by confirmatory TPSBx for entry into our AS programme (September 2009 to September 2010). These same men underwent a further staged TPSBx 24 months later, unless if there were clinical reasons to perform biopsy earlier such as a rising PSA. As part of our AS programme, these men were monitored with six monthly PSA, annual DRE, and a TPSBx every 24 months. TPSBx was performed under general anaesthesia with antibiotic prophylaxis. Men were placed in the lithotomy position and the scrotum was displaced upwards to expose the perineum. Transrectal ultrasound and a brachytherapy template were used to preferentially target the peripheral zone with core biopsies taken systematically on both sides from the anterior, mid, posterior, and basal sectors [5]. Around 24-38 cores were taken depending on prostate volume. A total of 24 cores were taken if prostate volume was $<30 \mathrm{~mL}$, four from the anterior, mid and posterior sectors. If the prostate volume was between $30-50 \mathrm{~mL}$, four additional cores were taken from the basal sector. Around 38 cores were taken if prostate volume was $>50 \mathrm{~mL}$, five from the anterior, mid, and posterior sectors, and four from the basal sector. A single practitioner performed all biopsies. Pathological characteristics, biopsy time, and complication data were collected from medical records. Erectile function was assessed with the International Index of Erectile Function-5 (IIEF-5) questionnaire prior to each biopsy and then at one, three, and six months following both initial and subsequent TPSBx. The degree of ED was stratified into five groups, based on IIEF-5 score [10]. Men were classified with ED if IIEF-5 score was less than 22. Two men were excluded from analysis of IIEF-5 as they reported no sexual activity (IIEF-5 = 0).

Paired t-test were performed to evaluate difference in IIEF-5. All statistical analyses were performed using SAS 9.4.

\section{Results}

Patients' demographics and biopsy Gleason scores are summarised in Table 1. Mean age at baseline TPSBx was 64.8 years $( \pm 5.9)$ and mean PSA at baseline was $9.2( \pm 6.3)$. The majority of men $(52 \%)$ presented with T2 disease. The most common Gleason score at first TPSBx was $3+3(36 \%), 3+4(25 \%), 4+3 / 4+5(9 \%)$, and benign (30\%). At second TPSBx, Gleason scores were 3+3 $(25 \%), 3+4(11 \%)$, $4+3 / 4+4 / 4+5(17 \%)$, and benign (45\%). Table 2 stratifies the degree of ED at baseline and postbiopsy.

With the first TPSBx, there was a statistically significant difference between the IIEF-5 score pre-TPSBx and one month post-TPSBx (Table 3, $\mathrm{P}<0.001$ ). Moreover, there was a statistically significant difference in IIEF-5 score between pre-TPSBx and three months postTPSBx $(P=0.001)$. At six months post-TPSBx, there was no statistically significant difference anymore with the IIEF-5 score pre-TPSBx $(P=0.681)$.

With the second TPSBx, there was a statistically significant difference between the IIEF-5 score pre-TPSBx and at one, three, and six months post-TPSBx (Table $3, \mathrm{P}<0.05$ ). There was a statistically significant difference in IIEF-5 score difference between the two biopsies, at three and six months post-TPSBx (Table 4, P <0.05). 
Table 1. Patient characteristics $(N=64)$.

\begin{tabular}{|l|c|}
\hline \multicolumn{1}{|l|}{} & $\mathbf{N}(\%)$ \\
\hline Mean Age, years (SD) & $63.4(5.9)$ \\
\hline Mean PSA, ng/mL (SD) & $9.2(6.3)$ \\
\hline DRE & $23(35.9)$ \\
\hline T1C & $33(51.6)$ \\
\hline T2 & $8(12.5)$ \\
\hline T3 & \\
\hline Biopsy 1 Gleason Score & $19(29.7)$ \\
\hline Benign & $23(35.9)$ \\
\hline $3+3$ & $16(25.0)$ \\
\hline $3+4$ & $5(7.8)$ \\
\hline $4+3$ & $1(1.6)$ \\
\hline $4+5$ & \\
\hline Biopsy 2 Gleason Score & $29(45.3)$ \\
\hline Benign & $16(25.0)$ \\
\hline $3+3$ & $7(10.9)$ \\
\hline $3+4$ & $4(6.3)$ \\
\hline $4+3$ & $2(3.1)$ \\
\hline $4+4$ & $5(7.8)$ \\
\hline $4+5$ & $1(1.6)$ \\
\hline Missing & $4.0(0.9)$ \\
\hline Mean operative time 1, mins (SD) & $6.0(1.3)$ \\
\hline Mean operative time 2, mins (SD) & \\
\hline
\end{tabular}

Table 2. Baseline and postbiopsy International Index Erectile Function-5 Score.

\begin{tabular}{|l|c|c|c|c|}
\hline & N at pre-TPSBx & $\begin{array}{c}\text { N at one month } \\
\text { post-TPSBx }\end{array}$ & $\begin{array}{c}\text { N at three months } \\
\text { post-TPSBx }\end{array}$ & $\begin{array}{c}\text { N at six months } \\
\text { post-TPSBx }\end{array}$ \\
\hline First TP & $29(45.3 \%)$ & 0 & $20(31.3 \%)$ & $29(45.3 \%)$ \\
\hline Without ED (IIEF-5 22-25) & $23(35.9 \%)$ & $12(18.8 \%)$ & $29(45.3 \%)$ & $23(35.9 \%)$ \\
\hline Mild ED (IIEF-5 17-21) & $3(4.7 \%)$ & $9(14.1 \%)$ & $5(7.8 \%)$ & $4(6.3 \%)$ \\
\hline Mild/Moderate ED (IIEF-5 12-16) & $5(7.8 \%)$ & $23(35.9 \%)$ & $7(10.9 \%)$ & $6(9.4 \%)$ \\
\hline Moderate ED (IIEF-5 8-11) & $2(3.1 \%)$ & $18(28.1 \%)$ & 0 & 0 \\
\hline Severe ED (IIEF-5 1-7) & $2(3.1 \%)$ & $2(3.1 \%)$ & $2(3.1 \%)$ & $2(3.1 \%)$ \\
\hline No sexual function (IIEF-5 0) & 0 & 0 & $1(1.6 \%)$ & 0 \\
\hline Missing & \multicolumn{5}{|l|}{} & $5(7.8 \%)$ & $12(18.7 \%)$ \\
\hline Second TP & $13(20.3 \%)$ & $3(4.7 \%)$ & $25(39.1 \%)$ & $24(37.5 \%)$ \\
\hline Without ED (IIEF-5 22-25) & $27(42.2 \%)$ & $5(7.8 \%)$ & $12(18.8 \%)$ & $11(17.2 \%)$ \\
\hline Mild ED (IIEF-5 17-21) & $11(17.2 \%)$ & $9(14.1 \%)$ & $4(6.2 \%)$ & $8(12.5 \%)$ \\
\hline Mild/Moderate ED (IIEF-5 12-16) & $5(7.8 \%)$ & $7(10.9 \%)$ & $4(6.2 \%)$ & $1(1.6 \%)$ \\
\hline Moderate ED (IIEF-5 8-11) & $3(4.7 \%)$ & $25(39.1 \%)$ & $9(14.1 \%)$ & $8(12.5 \%)$ \\
\hline Severe ED (IIEF-5 1-7) & $5(7.8 \%)$ & $15(23.4 \%)$ & $5(7.8 \%)$ & 0 \\
\hline No sexual function (IIEF-5 0) & 0 & 0 & \\
\hline Missing &
\end{tabular}


Table 3. Baseline mean and difference in International Index Erectile Function-5 Score.

\begin{tabular}{|c|c|c|c|}
\hline & Mean (SD) & $\begin{array}{c}\text { Mean difference } \\
\text { (SD) compared } \\
\text { with pre-TPSBx - } \\
\text { P-value }\end{array}$ & $\begin{array}{c}\text { Mean difference } \\
\text { (SD) compared } \\
\text { with previous - } \\
\text { P-value }\end{array}$ \\
\hline \multicolumn{4}{|l|}{ First TP } \\
\hline pre-TPSBx & $19.5(6.11)$ & & \\
\hline One month post-TPSBx & $10.5(5.4)$ & $\begin{array}{c}9.0(0.8) \\
<0.001\end{array}$ & $\begin{array}{c}9.0(0.8) \\
<0.001\end{array}$ \\
\hline Three months post-TPSBx & $18.7(5.6)$ & $\begin{array}{c}0.8(1.7) \\
0.001\end{array}$ & $\begin{array}{c}-8.2(6.1) \\
<0.001\end{array}$ \\
\hline Six month post-TPSBx & $19.6(5.4)$ & $\begin{array}{c}-0.1(1.8) \\
0.681\end{array}$ & $\begin{array}{c}-0.9(1.3) \\
<0.001\end{array}$ \\
\hline \multicolumn{4}{|l|}{ Second TP } \\
\hline pre-TPSBx & $16.6(7.1)$ & & \\
\hline One month post-TPSBx & $7.3(6.9)$ & $\begin{array}{c}9.3(6.6) \\
<0.001\end{array}$ & $\begin{array}{c}9.3(6.6) \\
<0.001\end{array}$ \\
\hline Three months post-TPSBx & $13.8(7.6)$ & $\begin{array}{c}2.9(3.9) \\
<0.001\end{array}$ & $\begin{array}{c}-6.6(6.2) \\
<0.001\end{array}$ \\
\hline Six month post-TPSBx & $15.9(7.7)$ & $\begin{array}{c}0.8(2.0) \\
0.003\end{array}$ & $\begin{array}{c}-2.1(3.3) \\
<0.001\end{array}$ \\
\hline
\end{tabular}

Table 4. Difference in International Index Erectile Function-5 Score between two biopsies.

\begin{tabular}{|l|c|c|c|}
\hline & $\begin{array}{c}\text { Mean difference } \\
\text { for first } \\
\text { TPSBx (SD) }\end{array}$ & $\begin{array}{c}\text { Mean difference } \\
\text { for second } \\
\text { TPSBx (SD) }\end{array}$ & $\begin{array}{c}\text { Paired T-test } \\
\text { P-value }\end{array}$ \\
\hline Compared to pre-TPSBx & $9.0(0.8)$ & $9.3(6.6)$ & 0.637 \\
\hline One month post-TPSBx & $0.8(1.7)$ & $2.9(3.9)$ & $<0.001$ \\
\hline Three months post-TPSBx & $-0.1(1.8)$ & $0.8(2.0)$ & 0.012 \\
\hline Six months post-TPSBx & $9.0(0.8)$ & $9.3(6.6)$ & 0.637 \\
\hline Compared to previous measurement & $-6.6(6.2)$ & 0.046 \\
\hline One month post-TPSBx & $-8.2(6.1)$ & $-2.1(3.3)$ & 0.011 \\
\hline Three months post-TPSBx & $-0.9(1.3)$ &
\end{tabular}

Of those men with normal erectile function before the initial TPSBx (IIEF-5 22-25), three out of 29 (10\%) reported significant deterioration in their erectile function (IIEF-5 <17) six months post-second TPSBX.

Complications with TPSBx were rare. There was no clot retention reported after the first TPSBx. Five men (8\%) experienced an episode of clot retention requiring catheterisation following the second TPSBx, with four of these related to urinary tract infection. There was a single episode of acute urinary retention following the first TPSBx, and there were no episodes of sepsis requiring admission with either biopsies. 


\section{Discussion}

In our cohort of men, there was a significant short-term deterioration in erectile function at one and three months following first TPSBx, compared to pre-TPSBx levels, which resolved at six month follow-up. Following a second biopsy, there was a deterioration in erectile function at one, three, and six months post-TPSBx compared to pre-TPSBx levels.

The effect of serial TRBx on erectile function remains unclear. Fujita et al reported on a study with 152 men on AS. There was a significant reduction in SHIM (an analogue of the IIEF-5) score with serial TRBx, especially after three biopsies [4]. There was also a significant difference in men without ED at baseline progressing to ED at follow-up, mean $3.2( \pm 2.3)$ years later. Braun et al demonstrated a small reduction in IIEF-6 score in a cohort undergoing serial TRBx [3]. They concluded this change was partially attributable to age-related ED, and the length of time their men were on AS rather than on serial TRBx. Both studies investigated the association between erectile function and biopsy over several years, but did not report on effects in the short and medium-term immediately post-TRBx. Their findings are likely to represent multiple variables influencing erectile function, rather than just the effect of a prostate biopsy.

The significant short-term deterioration in erectile function at one and three months following first TPSBx in our study is similar to that previously reported by Akbal et al, which showed a transient increase in ED at one month post-TPSBx that returned to baseline at six months [11]; however this was not seen by Klein et al, who reported no significant difference in IIEF-5 scores at one week, one month, and three months post-TPSBx [12]. The mean IIEF-5 score in their cohort was 15.18 which is several points lower than both our group and the Akbal et al study, and this in turn may have influenced outcomes.

Pepe et al reported on erectile function in 100 men who underwent repeated TPSBx, and concluded that serial TPSBx did not significantly worsen erectile function in the short to medium-term [9]. In contrast, our data suggests that serial TPSBx increases the risk of ED at one, three, and six months postbiopsy. Their study failed to report on erectile function before and after initial TPSBx, and also the time difference between initial and subsequent TPSBx.

The cause of ED postbiopsy is likely to be multifactorial; a combination of organic aetiology and psychological factors. Serial TPSBx involve repeated invasive procedures, and this increases the likelihood of trauma and long-term damage. Zisman et al suggested that biopsy related ED may be because of neurovascular bundle damage or secondary trauma because of nerve compression from haematoma or oedema [13]. This has been supported by a recent study from Tuncel et al that demonstrated radiological changes in the prostate parenchyma and neurovascular bundle in men with ED postbiopsy [14]. In addition, periprostatic nerve block (PPNB) is a commonly used form of analgesia for prostate biopsy, and it is likely to be partly responsible for neurovascular bundle damage. PPNB is generally believed to be a possible cause of transient ED postbiopsy [15].

The psychological impact of requiring a biopsy, anxiety surrounding a prostate cancer diagnosis, waiting for disclosure of results, and reduced quality-of-life caused by short-term complications of biopsy are all likely to affect erectile function. Zisman et al reported that biopsy-related anxiety peaked just before men were given their results, and the anxiety continued at one month postbiopsy in $64 \%$ of confirmed prostate cancer diagnosis [13]. Wade et al reported that men who complained of postbiopsy complications such as pain, haematuria, and haematospermia as a moderate/major problem had much higher levels of anxiety at one week postbiopsy compared to those who reported symptoms as minor/not problematic [16]. At 35 days postbiopsy, this anxiety was noticeably reduced. This decrease in anxiety over time may partially explain why IIEF-5 scores in our men recover towards prebiopsy baseline between one and three months follow-up. Anxiety and distress are generally not perceived to be associated with management of prostate cancer with AS, [17, 18] and the levels of both are generally favourable compared to anxiety and distress linked to radical treatment [19].

The Massachusetts Male Ageing Study (MMAS) and the Florey Adelaide Male Ageing Study both highlighted incidence of ED increasing with age [20, 21], with the MMAS reporting 26 cases per 1000 men annually in a cohort of men aged 40-69 years, an age range likely to mirror a typical AS group. A strength of our study was therefore to limit the effect of age by assessing erectile function immediately before and after TPSBx, rather than measuring erectile function at baseline and at a fixed follow-up point. Better data on the incidence of comorbidities in our cohort, especially given the strong correlation between LUTS and ED [22] would have helped to distinguish men who may have been predisposed to ED because of their pre-existing comorbidities. Our data was collected immediately before and after each TPSBx to ensure that any potential ED was likely to be linked solely to the TPSBx, limiting the influence of pre-existing comorbidities. 


\section{Conclusion}

Our AS protocol specifies a TPSBx every 24 months. An initial TPSBx can cause significant short-term ED, which generally recovers. Our study has shown that there is an increased risk of long-term ED with repeated TPSBx, and this should be considered against disease risk when considering further TPSBx.

The evidence for prostate magnetic resonance imaging (MRI) in selection and monitoring of patients for AS is increasing [23-25], and the use of serial imaging, PSA, and DRE to quantify possible disease progression may be helpful in the consideration of further TPSBx.

This risk of ED should be discussed and highlighted in the consent process.

\section{Conflicts of interest}

The author(s) declare that they have no conflicts of interest.

\section{References}

1. Dall'Era MA et al (2012) Active surveillance for prostate cancer: a systematic review of the literature Euro Urol 62(6) $976-83$ DOI: $10.1016 /$ j.eururo.2012.05.072

2. Parker C (2004) Active surveillance: towards a new paradigm in the management of early prostate cancer Lancet Oncol 5(2) 101-6 DOI: 10.1016/S1470-2045(04)01384-1 PMID: 14761814

3. Braun $\mathrm{K}$ et al (2014) Effect of repeated prostate biopsies on erectile function in men on active surveillance for prostate cancer J Urol 191(3) 744-9 DOI: 10.1016/i.juro.2013.08.054

4. Fujita K et al (2009) Serial prostate biopsies are associated with an increased risk of erectile dysfunction in men with prostate cancer on active surveillance $J$ Urol 182(6) 2664-9 DOI: 10.1016/j.juro.2009.08.044 PMID: 19836757

5. Vyas L et al (2014) Indications, results and safety profile of transperineal sector biopsies (TPSB) of the prostate: a single centre experience of 634 cases BJU Int 114(1) 32-7 DOI: 10.1111/bju.12282

6. Ayres BE et al (2012) The role of transperineal template prostate biopsies in restaging men with prostate cancer managed by active surveillance BJU Int 109(8)1170-6 DOI: 10.1111/j.1464-410X.2011.10480.x

7. Scott $S$ et al (2015) Is transperineal prostate biopsy more accurate than transrectal biopsy in determining final Gleason score and clinical risk category? A comparative analysis BJU Int 116(Suppl 3) 26-30. DOI: 10.1111/bju.13165 PMID: 26260531

8. Symons JL et al (2013) Outcomes of transperineal template-guided prostate biopsy in 409 patients BJU Int 112(5) 585-93 DOI: 10.1111/j.1464-410X.2012.11657.x PMID: 23551500

9. Pepe $\mathrm{P}$ et al (2013) Erectile function after repeat saturation prostate biopsy: our experience in 100 patients Arch Ital Urol Androl 85(3) 130-2 DOI: 10.4081/aiua.2013.3.130 PMID: 24085234

10. Rosen RC et al (1999) Development and evaluation of an abridged, 5-item version of the International Index of Erectile Function (IIEF-5) as a diagnostic tool for erectile dysfunction Int J Impot Res 11(6) 319-26 DOI: 10.1038/sj.ijir.3900472

11. Akbal C et al (2008) Erectile function in prostate cancer-free patients who underwent prostate saturation biopsy Europ Urol 53(3) 540-4 DOI: 10.1016/j.eururo.2007.06.039

12. Klein T et al (2010) The impact of prostate biopsy and periprostatic nerve block on erectile and voiding function: a prospective study J Urol 184(4) 1447-52 DOI: 10.1016/j.juro.2010.06.021 PMID: 20727540 
13. Zisman A et al (2001) The impact of prostate biopsy on patient well-being: a prospective study of pain, anxiety and erectile dysfunction J Urol 165(2) 445-54 DOI: 10.1097/00005392-200102000-00023 PMID: 11176394

14. Tuncel A et al (2014) Impact of transrectal prostate needle biopsy on erectile function: results of power Doppler ultrasonography of the prostate Kaohsiung J Med Sci 30(4)194-9 DOI: 10.1016/j.kjms.2013.11.004 PMID: 24656160

15. Glaser AP, Novakovic K and Helfand BT (2012) The impact of prostate biopsy on urinary symptoms, erectile function, and anxiety Curr Urol Rep 13(6) 447-54 DOI: 10.1007/s11934-012-0277-6 PMID: 22935886

16. Wade $\mathrm{J}$ et al (2013) Psychological impact of prostate biopsy: physical symptoms, anxiety, and depression J Clin Onco/ 31(33) 4235-41 DOI: $10.1200 / J C 0.2012 .45 .4801$ PMID: 24145343

17. van den Bergh RC et al (2010) Do anxiety and distress increase during active surveillance for low risk prostate cancer? J Urol 183(5) 1786-91 DOI: 10.1016/j.juro.2009.12.099

18. Venderbos LD et al (2014) A longitudinal study on the impact of active surveillance for prostate cancer on anxiety and distress levels Psychooncology 24(3) 348-54 DOI: 10.1002/pon.3657 PMID: 25138075

19. van den Bergh RC et al (2009) Anxiety and distress during active surveillance for early prostate cancer. Cancer 115(17) 3868-78 DOI: $10.1002 / \mathrm{cncr} .24446$ PMID: 19637245

20. Johannes CB et al (2000) Incidence of erectile dysfunction in men $\mathbf{4 0}$ to $\mathbf{6 9}$ years old: longitudinal results from the Massachusetts male aging study J Urol 163(2) 460-3 DOI: 10.1016/S0022-5347(05)67900-1 PMID: 10647654

21. Martin SA et al (2014) Predictors of sexual dysfunction incidence and remission in men J Sex Med 11(5) 1136-47 DOI: $10.1111 /$ jsm.12483 PMID: 24548342

22. McVary K (2006) Lower urinary tract symptoms and sexual dysfunction: epidemiology and pathophysiology BJU Int 97(Suppl 2) 23-28; discussion 44-25 DOI: 10.1111/j.1464-410X.2006.06102.x PMID: 16507050

23. Hoeks CM et al (2014) Value of 3-T multiparametric magnetic resonance imaging and magnetic resonance-guided biopsy for early risk restratification in active surveillance of low-risk prostate cancer: a prospective multicenter cohort study Invest Radiol 49(3)165-172 DOI: 10.1097/RLI.0000000000000008

24. Park BH et al (2014) Role of multiparametric 3.0-Tesla magnetic resonance imaging in patients with prostate cancer eligible for active surveillance BJU Inter 113(6) 864-70 DOI: 10.1111/bju.12423

25. Morgan VA et al (2011) Diffusion-weighted magnetic resonance imaging for monitoring prostate cancer progression in patients managed by active surveillance $B r J$ Radiol 84(997) 31-7 DOI: $10.1259 / \mathrm{bj} / 14556365$ 